\title{
A Robust Pre-Filter and Power Loading Design for Time Reversal UWB Systems over Time-Correlated MIMO Channels
}

\author{
Sajjad Alizadeh, Hossein Khaleghi Bizaki and Reza Saadat
}

\begin{abstract}
Conventional Time Reversal (TR) technique suffers from performance degradation in time varying Multiple-Input Multiple-Output Ultra-Wideband (MIMO-UWB) systems due to outdating Channel State Information (CSI) over time progressions. That is, the outdated CSI degrades the TR performance significantly in time varying channels. The correlation property of time correlated channels can improve the TR performance against other traditional TR designs. Based on this property, at first, we propose a robust TR-MIMO-UWB system design for a time-varying channel in which the CSI is updated only at the beginning of each block of data where the CSI is assumed to be known. As the channel varies over time, pre-processor blindly pre-equalizes the channel during the next symbol time by using the correlation property. Then, a novel recursive power allocation strategy is derived over time-correlated time-varying TR-MIMO-UWB channels. We show that the proposed power loading technique, considerably improves the Bit Error Rate (BER) performance of TR-MIMO-UWB system in imperfect CSI with robust pre-filter. The proposed algorithms lead to a cost-efficient CSI updating procedure for the TR optimization. Simulation results are provided to confirm the new design performance against traditional method.
\end{abstract}

Keywords-Time Reversal (TR) technique, MIMO channel, Ultra-Wideband (UWB) system, Temporal Correlation, Imperfect CSI, MMSE pre-equalizer.

\section{INTRODUCTION}

Ultra-wide band (UWB) communication systems have recently received much interest from both research community and industry [1]-[2]. However, due to the wide bandwidth property, UWB systems suffer from a very long delay spread of the multipath channels in indoor environments. Different types of the receiver structure such as RAKE, Transmitted Reference or the Decision Feedback Autocorrelation receivers can be implied [3]-[5] in which each technique has different complexities. To reduce the receiver complexity, non-coherent scheme is developed to bypass the complicated treatments on the UWB channel [6]-[7]. Accordingly, based on the idea of moving the complexity from the receiver to the transmitter, the Time Reversal (TR) preprocessing technique also referred to as the full pre-RAKE combining, which is originated from underwater acoustics and ultra sonic system [8], has been extended to the UWB applications. As a result, the TR technique can

Sajjad Alizadeh and Reza Saadat are with the Department of Electrical and Computer Engineering, Yazd University, Yazd, 89195-741, Iran, (e-mail: s.alizadeh@stu.yazd.ac.ir, rsaadat@yazd.ac.ir).

Hossein Khaleghi Bizaki is with the Electrical and Electronic Engineering University Complex, Malek Ashtar University of Technology, Tehran, Iran, (e-mail: bizaki@ee.iust.ac.ir).

Manuscript received March 14, 2014; revised April 28, 2014. be used to reduce the long delay spread of the UWB channel. Multiple-Input Multiple-Output (MIMO) solution, employing multiple antennas at the transmitter and receiver, is capable of increasing the data transmission rate by the spatial multiplexing without expanding the bandwidth and power [9]. In order to transmit parallel data streams simultaneously (spatial multiplexing), the Multi-Stream Interference (MSI) of the MIMO channel must be mitigated. The TR solution in the MIMO-UWB systems can mitigate not only the Inter-Symbol Interference (ISI) but also the MSI caused by transmitting several data streams, simultaneously [10]. The main bottleneck in the TR implementation is the necessity of the CSI at the transmitter, because in the TR technique we use the timereversed version of the estimated CSI as pre-filter. In practice, perfect CSI is not available in transmitter side due to the channel estimation error in time varying channels. In spite of TR's good performance, it is very sensitive to erroneous CSI due to imperfect channel estimation especially in time varying channel. Recently, the TR-based UWB system and its variations have been investigated in some literatures. We have sorted them into two categories:

First category of the researches on the TR pre-coding assumes that perfect CSI is given at the transmitter side [11][15]. Authors of [11] proposed an antenna selection scheme for the TR-MIMO-UWB communication system in a perfect CSI case to reduce the number of transmit antenna. Also, a Minimum Mean Square Error (MMSE) equalizer is used for the TR-UWB system with perfect CSI in [12] to mitigate the residual ISI and increase the transmission data rate. An ultra-high data rate TR-MIMO-UWB system with space-time pre-coding has been proposed in [13] based on the ZeroForcing (ZF) criterion in which the imperfect CSI has not been considered. Similar solution has been provided in [14] with the assumption of the spatial correlation among transmit and receive antenna. Also, the impact of spatial correlation on the TR-MIMO-UWB system has been investigated in [15] where several data streams can be transmitted by using only one antenna in a system named virtual TR-MIMO-UWB. Unfortunately, these methods suffer from the effects of the imperfect CSI in the transmitter, because the time variant channels and imperfect CSI have not been taken into account in these literatures.

Second category of the researches on the TR pre-coding assumes that imperfect CSI is available at the transmitter side [10], [16]-[22]. The effect of channel imperfection on the TRMIMO-UWB system performance has been evaluated in [10] 
for low and high data rate transmissions. Unfortunately, an optimization scheme has not been provided in this literature to overcome the effects of the channel imperfection. In [16]-[19], we had provided the pre-filtering solutions for the channel estimation error compensation in which the time variant channel is not considered. Especially, a power allocation scheme was proposed in [18] to improve the robust pre-filtered TRMIMO-UWB systems in an imperfect CSI for the quasi-static channels. On the other hand, the robustness of TR technique in imperfect CSI caused by a time varying channel environment has been studied in [20] by experimental results. It has been shown that, if the channel maintains some partial correlation with its previous ensembles, the TR method give a better performance even if the total correlation of the channels is very low. Authors of [20] gave to us an idea to develop our previous works [18] (pre-filtering and power loading in the quasistatic TR-MIMO-UWB systems) for the temporal correlated time varying TR-MIMO channels in an imperfect CSI. Also, a post-time-reversed MIMO-UWB transmission scheme has been proposed in [21] which improves the TR robustness against imperfect CSI caused by channel estimation error when compared with the conventional TR scheme. Finally, an analysis without any improvement solution has been provided in [22] on the effect of the channel estimation error on the performance of the TR-UWB systems.

As it is observed from previous literatures, all of them assume the quasi-static channels in their analysis. In order to increase the TR performance in time varying channels, the preprocessors should be optimized approximately in some symbol duration [23]. On the other hand, the generation of reliable channel feedback in some short symbol time is complicated in time-varying channel and can be led to high bandwidth overhead. From practical implementation point of view, most of the TRs have been designed assuming that the wireless channel can be regarded as constant over a block of data. In mobile applications where the channel is time-varying, the assumption that the channel is constant over some periods only holds approximately and will affects the TR performance that are designed based on this assumption. Hence, a judicious and innovative TR system design that takes the time-varying nature of the channel into account is the key to solve the above problems.

Based on mentioned researches on the TR pre-coding and also, works of [18] and [23], we extend and unify the TR concepts to time varying MIMO channels in an imperfect CSI, which will allow us to improve mobile systems performance, as well as to provide guidelines for future precoder designs employing low feedback overhead. On the other hand, a recursive power allocation strategy is proposed to more improve the performance of the proposed robust TRMIMO-UWB system. According to authors best knowledge, no research has been reported so far about time varying and time correlated TR-MIMO-UWB systems. Therefore, it is essential to analyze and improve the TR-MIMO-UWB systems in mobile applications with imperfect CSI. Our current works can be used for the mobile communication networks, such as Ad Hoc networks and so on. The rest of the paper is organized as follows; we introduce the system model in Section II.
A novel solution based on a MMSE criterion and a novel recursive power allocation scheme are derived for TR-MIMOUWB system over time varying channels in Section III and IV, respectively. Numerical and simulation results characterizing the performance of the proposed methods are presented in Section V, and finally, conclusions are drawn in Section VI.

\section{System Description In TIME CorRelated CHANNELS}

A TR-MIMO-UWB communication system includes a transmitter equipped with antennas and a receiver equipped with antennas is depicted in Fig. 1. Let us consider an impulsive (Impulse Radio) UWB (IR-UWB) system using Binary Pulse Amplitude Modulation (BPAM) with pulse shaping according to the Federal Communications Commission (FCC) desired power spectral density [1]-[2]. The transmitted signal for every input of the MIMO system is represented as,

$$
x(t)=\sqrt{E_{b}} \sum_{k=-\infty}^{+\infty} d_{k} p(t-k T)
$$

where $d_{k}=\{ \pm 1\}$ is the binary transmit symbol, $E_{b}$ is the bit energy, $p(t)$ is the desired pulse shape, and $T$ denotes the symbol duration. According to Fig. 1, at first, the input signal is converted into $N_{r}$ streams, pre-equalized with an optimized MMSE pre-equalizer, pre-coded with TR pre-filter, and then sent to $N_{r}$ transmitting antennas, simultaneously. The resultant signal passes through the multipath MIMO channel and then, is corrupted by an Additive White Gaussian Noise (AWGN). Thus, there are $N_{t} \times N_{r}$ multipath channel between transmit and receive antennas. For simplicity of our analysis, we use the tapped delay line (TDL) model with maximum $L_{g}$ taps [10]-[11]. If $\tau_{d}$ and $T$ are the delay spread of the channel and the symbol duration, respectively, then $L_{g} \cong \frac{\tau_{d}}{T}$. In fact, we use a TDL model with equal tap spacing of $T$. Note that in our application, $\tau_{d} \gg T$ then we have a frequencyselective model for each multipath channel between transmit and receive antennas. Furthermore, because of using the very short duration pulses, IR-UWB meets extremely resolvable multipath components (MPC) leading to long delay spread $\tau_{d}$, in that, two multipath components (taps) with delays $\tau_{m}$ and $\tau_{n}$ are resolvable if $\left|\tau_{m}-\tau_{n}\right| \geq T$. We assume $\left|\tau_{m}-\tau_{n}\right| \geq T$. Also, the UWB channels are assumed to be time correlated across blocks [23]. The TR pre-filter is used in the spatial multiplexing UWB system in order to cope with the ISI and MSI problems. Since the ultra-high data rate UWB transmission usually requires extremely short symbol time, thus ISI and MSI are very strong. These interferences, especially MSI, are not absolutely eliminated by TR pre-filter [13]. Therefore, to completely eliminate the residual ISI and MSI, a MMSE pre-equalizer, i.e. $\mathbf{F}_{M M S E}$ in Fig. 1, is needed in transmitter as will be considered in Section 3. Both MMSE pre-equalizer and TR pre-filter require the perfect CSI. The CSI can be estimated using the training symbols. The training pattern employed for channel estimation is similar to what is considered in [24], in which at the beginning of every block, the first $N_{t}$ symbols are assigned as training sequence, once in every $T_{f}=N T$ seconds, where every block includes 


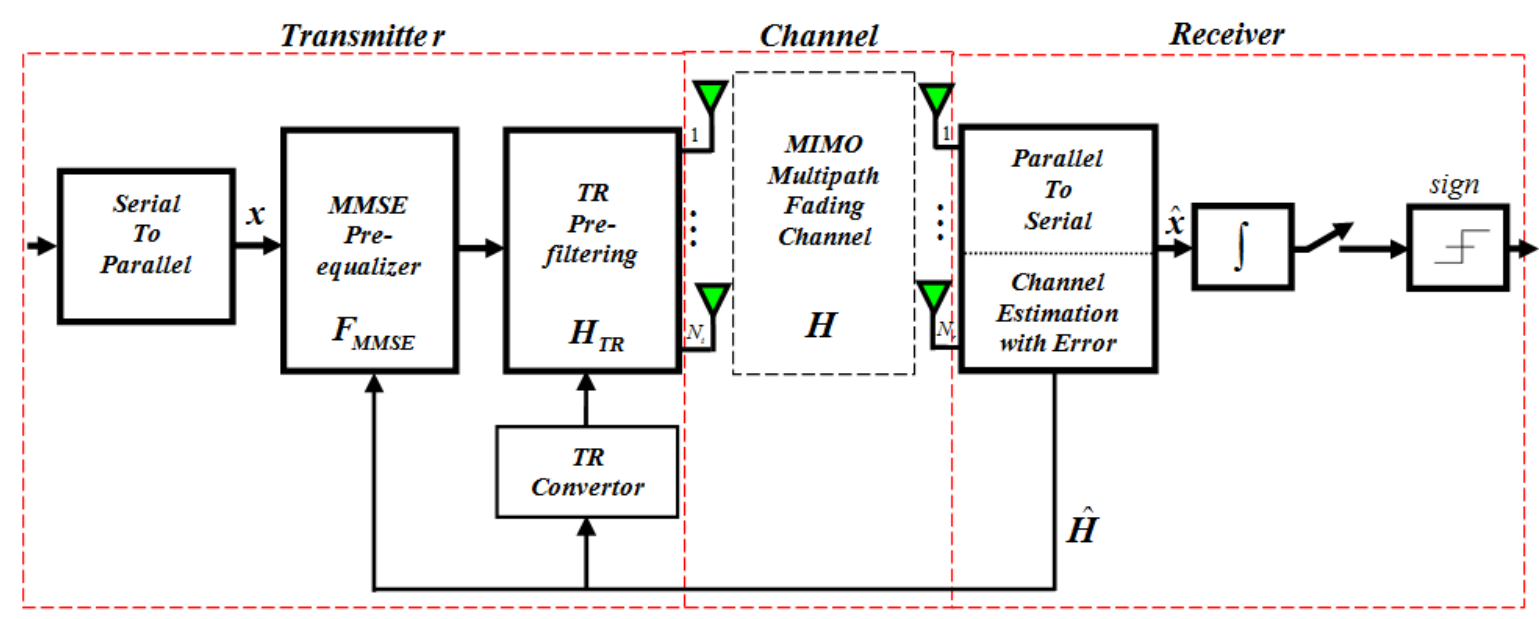

Fig. 1. TR-MIMO-UWB system model.

$N$ symbols. As in [24], the MMSE channel estimation is assumed in this paper. Then, the estimated CSI is fedback to transmitter through the feedback channel. In practice, perfect CSI is not available in the transmitter side due to non-ideal channel estimator. Pre-processors (TR pre-filtering and MMSE pre-equalizing) are influenced by the imperfect CSI effects. Finally, the data stream is detected based on a simple integrateand-dump receiver with the sampling rate of $\frac{1}{T}$.

The effect of channel estimation error in a time varying channel can be considered as $\mathbf{H}_{t}=\hat{\mathbf{H}}_{t}+\boldsymbol{\Delta} \mathbf{H}_{t}$ where $\mathbf{H}_{t}, \hat{\mathbf{H}}_{t}$ and $\Delta \mathbf{H}_{t}$ are the true value, estimated value and estimation error of the CIR at the time instant $t$, respectively. It is assumed that the entries of $\Delta \mathbf{H}_{t}$ are i.i.d. random variables with zero mean complex Gaussian noise. The estimated value $\hat{\mathbf{H}}_{t}$ is converted to the TR pre-filter, as $\mathbf{H}_{T R, t}$, by the TR convertor. This function will be exactly explained after some following basic definitions in next paragraphs of this section. The estimated CIR between $i^{\text {th }}$ transmit antenna and $j^{\text {th }}$ receive antenna $\left(i=1, \ldots, N_{t}, \quad j=1, \ldots, N_{r}\right)$ at the time instant $t$ can be denoted as,

$$
\hat{h}_{i j, t}(t)=\sum_{l=0}^{L_{g}-1} \hat{\alpha}_{l}^{i j, t} \delta\left(t-\hat{\tau}_{l}^{i j, t}\right)
$$

where $\hat{\alpha}_{l}$ is the estimated fading coefficient of the $l^{\text {th }}$ multipath components (MPC), $\hat{\tau}_{l}$ is the corresponding delay of the $l^{\text {th }}$ tap and $L_{g}$ is the number of resolvable MPCs. The discrete time vector form of CIR in reversed order at the time instant $t$ is denoted as: $\hat{\mathbf{h}}_{i j, t}=\left[\hat{h}_{i j, t}^{L_{g}-1}, \cdots, \hat{h}_{i j, t}^{l}, \cdots, \hat{h}_{i j, t}^{0}\right]_{1 \times L_{g}}$ where $\hat{h}_{i j, t}^{l}$ is the amplitude of the $l^{\text {th }}$ path for the channel between $i^{\text {th }}$ transmit antenna and $j^{\text {th }}$ receive antenna at time instant $t$. Also, the estimation error vector of the CIR is defined as: $\boldsymbol{\Delta} \mathbf{h}_{i j, t}=\left[\Delta h_{i j, t}^{L_{g}-1}, \cdots, \Delta h_{i j, t}^{l}, \cdots, \Delta h_{i j, t}^{0}\right]_{1 \times L_{g}}$ where $i=1, \ldots, N_{t}, j=1, \ldots, N_{r}$ and $l=0, \ldots, L_{g}-1$. The probability distribution of the error coefficients are considered as: $\Delta h_{i j, t}^{l} \sim C N\left(0, \sigma_{e}^{2}\right)$ where $\sigma_{e}^{2}$ is the estimation error variance. As in [10]-[11], the estimated CIR and estimation error matrices of size in the time instant $t$, can be written as:

$$
\begin{aligned}
& \hat{\mathbf{H}}_{t}=\left(\begin{array}{llll}
\hat{\mathbf{h}}_{11, t} & \hat{\mathbf{h}}_{21, t} & \cdots & \hat{\mathbf{h}}_{N_{t} 1, t} \\
\hat{\mathbf{h}}_{12, t} & \hat{\mathbf{h}}_{22, t} & \cdots & \hat{\mathbf{h}}_{N_{t} 2, t} \\
\vdots & \vdots & \vdots & \vdots \\
\hat{\mathbf{h}}_{1 N_{r}, t} & \hat{\mathbf{h}}_{2 N_{r}, t} & \cdots & \hat{\mathbf{h}}_{N_{t} N_{r}, t}
\end{array}\right) \\
& \boldsymbol{\Delta} \mathbf{H}_{t}=\left(\begin{array}{llll}
\boldsymbol{\Delta} \mathbf{h}_{11, t} & \boldsymbol{\Delta} \mathbf{h}_{21, t} & \cdots & \boldsymbol{\Delta} \mathbf{h}_{N_{t} 1, t} \\
\boldsymbol{\Delta} \mathbf{h}_{12, t} & \boldsymbol{\Delta} \mathbf{h}_{22, t} & \cdots & \boldsymbol{\Delta} \mathbf{h}_{N_{t} 2, t} \\
\vdots & \vdots & \vdots & \vdots \\
\boldsymbol{\Delta} \mathbf{h}_{1 N_{r}, t} & \boldsymbol{\Delta} \mathbf{h}_{2 N_{r}, t} & \cdots & \boldsymbol{\Delta} \mathbf{h}_{N_{t} N_{r}, t}
\end{array}\right)
\end{aligned}
$$

Note that the size of matrices $\hat{\mathbf{H}}_{t}$ and $\boldsymbol{\Delta} \mathbf{H}_{t}$ is $N_{r} \times N_{t} L_{g}$. Also, $\mathbf{C}_{\boldsymbol{\Delta} \mathbf{H}_{t}}=E\left\{\boldsymbol{\Delta} \mathbf{H}_{t} \boldsymbol{\Delta} \mathbf{H}_{t}^{H}\right\}$ is the estimation error covariance matrix and $E\left\{\boldsymbol{\Delta} \mathbf{H}_{t}\right\}=0$ is assumed. For simplicity of analysis, we assume that the estimation errors of the different sub-channels are statistically independent, that is, they have a common error variance $\sigma_{e}^{2}$. Thus, we have $\mathbf{C}_{\boldsymbol{\Delta} \mathbf{H}_{t}}=E\left\{\boldsymbol{\Delta} \mathbf{H}_{t} \boldsymbol{\Delta} \mathbf{H}_{t}^{H}\right\}=\sigma_{e}^{2} \mathbf{I}$ where $\mathbf{I}$ is the identity matrix. If the estimated CIR of all sub-channels, i.e. $\hat{\mathbf{h}}_{i j, t}$, are known at the transmitter side, time reversed version of them are used to TR pre-filter to pre-equalize the transmitted data. Thus, we can construct the pre-filter matrix based on the time reversed form of the estimated sub-channels by the TR convertor which is a $N_{t} L_{g} \times N_{r}\left(2 L_{g}-1\right)$ matrix in [10]-[11] as

$$
\mathbf{H}_{T R, t}=\left(\begin{array}{llll}
\overline{\mathbf{H}}_{11, t} & \overline{\mathbf{H}}_{12, t} & \cdots & \overline{\mathbf{H}}_{1 N_{r}, t} \\
\overline{\mathbf{H}}_{21, t} & \overline{\mathbf{H}}_{22, t} & \cdots & \overline{\mathbf{H}}_{2 N_{r}, t} \\
\vdots & \vdots & \vdots & \vdots \\
\overline{\mathbf{H}}_{N_{t} 1, t} & \overline{\mathbf{H}}_{N_{t} 2, t} & \cdots & \overline{\mathbf{H}}_{N_{t} N_{r}, t}
\end{array}\right)
$$

where each sub-matrix $\overline{\mathbf{H}}_{i j, t}$ is an $L_{g} \times\left(2 L_{g}-1\right)$ Toeplitz matrix defined by [10]-[11] as

$$
\begin{aligned}
& \overline{\mathbf{H}}_{i j, t}= \\
& \left(\begin{array}{lllllll}
\hat{h}_{i j, t}^{0} & \cdots & \hat{h}_{i j, t}^{L_{g}-1} & 0 & \cdots & & 0 \\
0 & \hat{h}_{i j, t}^{0} & \cdots & \hat{h}_{i j, t}^{L_{g}-1} & 0 & \cdots & \vdots \\
\vdots & & \ddots & & \ddots & & 0 \\
0 & \cdots & 0 & \hat{h}_{i j, t}^{0} & \cdots & & \hat{h}_{i j, t}^{L_{g}-1}
\end{array}\right)
\end{aligned}
$$

In fact, for each sub-matrix $\overline{\mathbf{H}}_{i j, t}$, we have 
$\overline{\mathbf{H}}_{i j, t}=\operatorname{Toeplitz}\left(T R\left(\hat{\mathbf{h}}_{i j, t}\right)\right)$ where Toeplitz(.) and $T R($.$) are the Toeplitz matrix maker and the time$ reversed order of a vector, respectively. Therefore, the TR pre-filter by the TR convertor can be written as $\mathbf{H}_{T R, t}=\operatorname{Toeplitz}\left(T R\left(\hat{\mathbf{H}}_{t}^{H}\right)\right)=\mathcal{F}\left(\hat{\mathbf{H}}_{t}^{H}\right)$ where the TR operation is applied on the vectors $\hat{\mathbf{h}}_{i j, t}$ of the matrix $\hat{\mathbf{H}}_{t}^{H}$. Also, $(.)^{H}$ denotes the complex conjugate transpose of the matrix $\hat{\mathbf{H}}_{t}$. The equivalent TR channel response matrix of size $N_{r} \times N_{r}\left(2 L_{g}-1\right)$ is given by

$$
\mathbf{H}_{t} \mathbf{H}_{T R, t}=\left(\begin{array}{llll}
\overline{\mathbf{h}}_{11, t} & \overline{\mathbf{h}}_{21, t} & \cdots & \overline{\mathbf{h}}_{N_{r} 1, t} \\
\overline{\mathbf{h}}_{12, t} & \overline{\mathbf{h}}_{22, t} & \cdots & \overline{\mathbf{h}}_{N_{r} 2, t} \\
\vdots & \vdots & \vdots & \vdots \\
\overline{\mathbf{h}}_{1 N_{r}, t} & \overline{\mathbf{h}}_{2 N_{r}, t} & \cdots & \overline{\mathbf{h}}_{N_{r} N_{r}, t}
\end{array}\right)
$$

where each sub-channel $\overline{\mathbf{h}}_{i j, t}$ is a $1 \times\left(2 L_{g}-1\right)$ autocorrelation $(i=j)$ or cross-correlation $(i \neq j)$ vector. The received symbols vector at the MIMO channel output with MMSE pre-equalizer and TR pre-filter can be written as $\hat{\mathbf{x}}_{t}=\mathbf{H}_{t} \mathbf{H}_{T R, t} \mathbf{F}_{M M S E, t} \mathbf{x}_{t}+\mathbf{n}_{t}$ where $\mathbf{x}_{t}, \hat{\mathbf{x}}_{t}$, and $\mathbf{n}_{t}$ are the transmitted symbols, the received symbols and AWGN, respectively. The vector $\mathrm{x}_{t}$ contains i.i.d. random variables with zero mean $E\left\{\mathbf{x}_{t}\right\}=\mathbf{0}$ and variance $E\left\{\mathbf{x}_{t} \mathbf{x}_{t}^{H}\right\}=\sigma_{\mathbf{x}}^{2} \mathbf{I}$ with antipodal modulation. Also, we assume $\mathbf{n}_{t} \sim C N\left(\mathbf{0}, \sigma_{\mathbf{n}}^{2} \mathbf{I}\right)$ where $\sigma_{\mathbf{n}}^{2}=E\left\{\mathbf{n}_{t} \mathbf{n}_{t}^{H}\right\}$. Summarily, the size of the input and output vectors or matrices of each block of Fig. 1 can be presented as follows; the transmitted and received symbol vectors $\mathbf{x}$ and $\hat{\mathbf{x}}$ as well noise vector $\mathbf{n}$ are of size $N_{r} \times 1$. Also, the MMSE pre-coding matrix $\mathbf{F}_{M M S E}$ and the TR pre-filter matrix $\mathbf{H}_{T R}$ are of size $N_{r}\left(2 L_{g}-1\right) \times N_{r}$ and $N_{t} L_{g} \times N_{r}\left(2 L_{g}-1\right)$, respectively. The true value $\mathbf{H}$ and the estimated value $\hat{\mathbf{H}}$ of the channel matrix are of size $N_{r} \times N_{t} L_{g}$. Therefore, the total pre-coding $\mathbf{H}_{T R} \mathbf{F}_{M M S E}$ is a $N_{t} L_{g} \times N_{r}$ matrix.

To analysis the TR-MIMO-UWB system in the time correlated channels, we define $\hat{\mathbf{H}}_{t-\tau}$ as the estimated CIR matrix at the time instant $t-\tau$. In fact, $\hat{\mathbf{H}}_{t-\tau}$ corresponds to the channel state $\tau$ seconds earlier than $\hat{\mathbf{H}}_{t}$. We assume that $\hat{\mathbf{H}}_{t}$ and $\hat{\mathbf{H}}_{t-\tau}$ are correlated realizations of the estimated channels. Thus, given the outdated CSI, i.e. $\hat{\mathbf{H}}_{t-\tau}$, we can characterize the unknown current CSI, i.e. $\hat{\mathbf{H}}_{t}$, as introduced in [23],

$$
\hat{\mathbf{H}}_{t}=\rho \hat{\mathbf{H}}_{t-\tau}+\sigma_{h} \sqrt{1-\rho^{2}} \mathbf{E}_{t}=\rho \hat{\mathbf{H}}_{t-\tau}+\Delta \mathbf{E}
$$

where $\sigma_{h}^{2}=E\left\{\left\|\hat{\mathbf{h}}_{i j, t}\right\|^{2}\right\}$ is the variance of the channel coefficients. Note that the different sub-channels are statistically independent assumed, that is, they have a common variance. The entries of the matrix $\mathbf{E}_{t}$ in (3) are assumed to be i.i.d. zero-mean complex Gaussian random variables, i.e. $\mathbf{E}_{t} \sim$ $C N(\mathbf{0}, \mathbf{I})$. Also, the matrix $\boldsymbol{\Delta} \mathbf{E}$ is the $C N\left(\mathbf{0}, \sigma_{h}^{2}\left(1-\rho^{2}\right) \mathbf{I}\right)$ distributed uncertainty on the true estimated channel given the outdated CSI, $\hat{\mathbf{H}}_{t-\tau}$. The time correlation coefficient $\rho$ between the time instants $t$ and $t-\tau$ is defined in [23] as,

$$
\rho=\frac{E\left\{\hat{\mathbf{h}}_{i j, t} \hat{\mathbf{h}}_{i j, t-\tau}^{H}\right\}}{\sqrt{E\left\{\left\|\hat{\mathbf{h}}_{i j, t}\right\|^{2}\right\} E\left\{\left\|\hat{\mathbf{h}}_{i j, t-\tau}\right\|^{2}\right\}}}
$$

The similar discussion can be presented for the estimation error matrix $\Delta \mathbf{H}_{t}$, i.e., we have,

$$
\boldsymbol{\Delta} \mathbf{H}_{t}=\rho \boldsymbol{\Delta} \mathbf{H}_{t-\tau}+\sigma_{e} \sqrt{1-\rho^{2}} \mathbf{E}_{t}^{\prime}=\rho \boldsymbol{\Delta} \mathbf{H}_{t-\tau}+\boldsymbol{\Delta} \mathbf{E}^{\prime}
$$

where $\mathbf{E}^{\prime}{ }_{t} \sim C N(\mathbf{0}, \mathbf{I})$ and $\boldsymbol{\Delta} \mathbf{E}^{\prime} \sim C N\left(\mathbf{0}, \sigma_{e}^{2}\left(1-\rho^{2}\right) \mathbf{I}\right)$. Because the matrix $\mathbf{H}_{T R, t}$ is a function of the matrix $\hat{\mathbf{H}}_{t}$ as $\mathbf{H}_{T R, t}=\mathcal{F}\left(\hat{\mathbf{H}}_{t}^{H}\right)$, it can be written as,

$$
\begin{aligned}
\mathbf{H}_{T R, t} & =\rho \mathbf{H}_{T R, t-\tau}+\sigma_{h_{e q}} \sqrt{1-\rho^{2}} \mathbf{E}_{t_{e q}} \\
& =\rho\left(\operatorname{Toeplitz}\left(T R\left(\hat{\mathbf{H}}_{t-\tau}^{H}\right)\right)\right)+\boldsymbol{\Delta} \mathbf{E}_{e q}
\end{aligned}
$$

where $\mathbf{E}_{t_{e q}} \sim C N(\mathbf{0}, \mathbf{I})$ and it is different from $\mathbf{E}_{t}$ in (3) and $\mathbf{E}_{t}^{\prime}$ in (5), even though they have the same statistical properties. Also, $\sigma_{h_{e q}}^{2}$ is the variance of the TR channel coefficients and $\boldsymbol{\Delta} \mathbf{E}_{e q} \sim C N\left(\mathbf{0}, \sigma_{h_{e q}}^{2}\left(1-\rho^{2}\right) \mathbf{I}\right)$. Finally, we define the conventional TR as the time reversal based MIMO-UWB system in an imperfect CSI without any optimizations. Also, the proposed pre-filter optimization means the application of the conventional TR (time reversal based MIMO-UWB system in an imperfect CSI) with the MMSE optimized pre-filter.

\section{Robust PRE-FILTER Optimization}

It is worthwhile to note that the interferences (ISI and MSI) are not absolutely eliminated by TR pre-filter. Especially in ultra-high data rate UWB transmission, we need to extremely short symbol in which leads to strong ISI and MSI. On the other hands, a TR pre-filter works in time domain to raise the SNR, but simultaneously leads to a BER performance floor caused by the ISI and MSI [13]. In this section, we derive a MMSE pre-equalization solution in the presence of the estimation error in time varying channels. The error vector that is needed to be considered for the system illustrated in Fig. 1 should be the difference between the transmitted symbols and the detected symbols, i.e.,

$$
\begin{aligned}
\mathbf{e}_{t} & =\hat{\mathbf{x}}_{t}-\mathbf{x}_{t} \\
& =\left(\left(\hat{\mathbf{H}}_{t}+\boldsymbol{\Delta} \mathbf{H}_{t}\right) \mathbf{H}_{T R, t} \mathbf{F}_{M M S E, t} \mathbf{x}_{t}+\mathbf{n}_{t}\right)-\mathbf{x}_{t}
\end{aligned}
$$

where the TR matrix $\mathbf{H}_{T R, t}$ is constructed based on $\hat{\mathbf{H}}_{t}$ as presented in Section II. The MMSE solution should minimize the cost function $E\left\{\left\|\mathbf{e}_{t}\right\|^{2}\right\}=E\left\{\left\|\hat{\mathbf{x}}_{t}-\mathbf{x}_{t}\right\|^{2}\right\}$ with respect to $\mathbf{F}_{M M S E, t}$. Therefore, the linear MMSE pre-equalizer can be obtained by solving of (8). Calculation of the conditional expectation, as required in (8), seems to be difficult. We consider an approximately solution to solve it. To do this, we use the correlation model presented in (3), (5) and (6). According to [23], this model describes the time correlation coefficient function of the estimated CSI between the time instants $t-\tau$ and $t$ as $\rho=J_{0}\left(2 \pi f_{d} \tau\right)$ in which $f_{d}$ is the maximum Doppler frequency. Also, $J_{0}($.$) denotes the zero order Bessel function$ and $\tau=k T ; k \leq N$ where $k$ denotes as number of symbol and $T$ is the data symbol duration. Knowledge of the Doppler spread is assumed in the design of the above proposal filter. Derivation of linear MMSE pre-equalization has been carried out in [25], Appendix E and also, an imperfect CSI based derivation has been provided in section 6.2.2 of [25]. Clearly, at first we take the conditional expectation over $\Delta \mathbf{H}_{t}, \boldsymbol{\Delta} \mathbf{E}$ and $\Delta \mathbf{E}_{e q}$ similar to [23], because of using conditional CSI 


$$
\begin{aligned}
& \mathbf{F}_{M M S E, t}=\arg \min _{\tilde{\mathbf{F}}_{M M S E, t}} E_{\hat{\mathbf{H}}_{t} \mid \hat{\mathbf{H}}_{t-\tau}}\left\{E_{\mathbf{H}_{T R, t} \mid \mathbf{H}_{T R, t-\tau}}\left\{E_{\mathbf{x}, \Delta \mathbf{H}_{t}}\left\{\left\|\left(\left(\hat{\mathbf{H}}_{t}+\boldsymbol{\Delta} \mathbf{H}_{t}\right) \mathbf{H}_{T R, t} \tilde{\mathbf{F}}_{M M S E, t} \mathbf{x}_{t}+\mathbf{n}_{t}\right)-\mathbf{x}_{t}\right\|^{2}\right\}\right\}\right\} \\
& \text { subject to } E_{\hat{\mathbf{H}}_{t} \mid \hat{\mathbf{H}}_{t-\tau}}\left\{E_{\mathbf{H}_{T R, t} \mid \mathbf{H}_{T R, t-\tau}}\left\{E_{\mathbf{x}, \Delta \mathbf{H}_{t}}\left\{\left\|\tilde{\mathbf{F}}_{M M S E, t} \mathbf{x}_{t}\right\|^{2}\right\}\right\}\right\}=\sigma_{\mathbf{x}}^{2} \\
& \mathbf{F}_{M M S E, t}=\rho^{2}\left(\hat{\mathbf{H}}_{t-\tau} \mathbf{H}_{T R, t-\tau}\right)^{H} \quad\left\{\rho^{4}\left(\hat{\mathbf{H}}_{t-\tau} \mathbf{H}_{T R, t-\tau}\right)\left(\hat{\mathbf{H}}_{t-\tau} \mathbf{H}_{T R, t-\tau}\right)^{H}\right. \\
& \left.+\mathbf{C}_{\Delta \mathbf{H}_{t}}+\mathbf{C}_{\Delta \mathbf{E}}+\mathbf{C}_{\Delta \mathbf{E}_{e q}}+\mathbf{C}_{\Delta \mathbf{E}, \Delta \mathbf{E}_{e q}}+\left(\frac{1}{S N R}\right) \mathbf{I}\right\}^{-1}
\end{aligned}
$$

model as described in (3), (5) and (6) similar to Equation (2) of [23], and then, substitute $\rho \hat{\mathbf{H}}_{t-\tau}, \rho \mathbf{H}_{T R, t-\tau}$ and the mutually covariance matrices $\mathbf{C}_{\Delta \mathbf{H}_{t}}, \mathbf{C}_{\Delta \mathbf{E}}, \mathbf{C}_{\Delta \mathbf{E}_{e q}}$ and $\mathbf{C}_{\Delta \mathbf{E}, \Delta \mathbf{E}_{e q}}$ as the channel imperfections in the Equation $6.24 \mathrm{~b}$ of [25]. Accordingly, we get the MMSE solution as (9) in which $S N R=\frac{\sigma_{\mathbf{x}}^{2}}{\sigma_{\mathbf{n}}^{2}}$ and $\mathbf{C}_{\Delta \mathbf{H}_{t}}$ is the channel estimation error covariance matrix as $\mathbf{C}_{\boldsymbol{\Delta}} \mathbf{H}_{t}=E_{\boldsymbol{\Delta} \mathbf{H}_{t}}\left\{\boldsymbol{\Delta} \mathbf{H}_{t} \boldsymbol{\Delta} \mathbf{H}_{t}^{H}\right\}=\sigma_{e}^{2} \mathbf{I}$ in which $\sigma_{e}^{2}$ is the common estimation error variance for all of the sub-channels. Note that we assumed $\Delta \mathbf{E}, \Delta \mathbf{H}_{t}$ and also, $\Delta \mathbf{E}_{e q}$ and $\Delta \mathbf{H}_{t}$ are statistically independent. Also, according to (6), $\hat{\mathbf{H}}_{t}$ and $\mathbf{H}_{T R, t}$ are statistically dependent. The covariance matrices can be obtained as,

$$
\begin{aligned}
& \mathbf{C}_{\boldsymbol{\Delta} \mathbf{E}}=E\left\{\boldsymbol{\Delta} \mathbf{E} \boldsymbol{\Delta} \mathbf{E}^{H}\right\}=\sigma_{h}^{2}\left(1-\rho^{2}\right) \mathbf{I} \\
& \mathbf{C}_{\boldsymbol{\Delta} \mathbf{E}^{\prime}}=E\left\{\boldsymbol{\Delta} \mathbf{E}^{\prime} \boldsymbol{\Delta} \mathbf{E}^{\prime H}\right\}=\sigma_{e}^{2}\left(1-\rho^{2}\right) \mathbf{I} \\
& \mathbf{C}_{\boldsymbol{\Delta}} \mathbf{E}_{e q}=E\left\{\boldsymbol{\Delta} \mathbf{E}_{e q} \boldsymbol{\Delta} \mathbf{E}_{e q}^{H}\right\}=\sigma_{h_{e q}}^{2}\left(1-\rho^{2}\right) \mathbf{I} \\
& \mathbf{C}_{\boldsymbol{\Delta} \mathbf{E}, \boldsymbol{\Delta} \mathbf{E}_{e q}}=E\left\{\boldsymbol{\Delta} \mathbf{E} \boldsymbol{\Delta} \mathbf{E}_{e q}^{H}\right\}=\sigma_{h} \sigma_{h_{e q}}\left(1-\rho^{2}\right) \mathbf{I}
\end{aligned}
$$

With respect to (5), $\mathbf{C}_{\boldsymbol{\Delta} \mathbf{H}_{t}}=\rho^{2} \mathbf{C}_{\boldsymbol{\Delta}} \mathbf{H}_{t-\tau}+\mathbf{C}_{\boldsymbol{\Delta} \mathbf{E}^{\prime}}$ in which $\mathbf{C}_{\boldsymbol{\Delta} \mathbf{H}_{t-\tau}}=E_{\boldsymbol{\Delta} \mathbf{H}_{t-\tau}}\left\{\boldsymbol{\Delta} \mathbf{H}_{t-\tau} \boldsymbol{\Delta} \mathbf{H}_{t-\tau}^{H}\right\}$ is the estimation error covariance matrix at the time instant $t-\tau$. If the perfect CSI is available (ideal channel estimation), Equation (9) is referred to as MMSE pre-filter optimization design in which $\mathbf{C}_{\boldsymbol{\Delta}} \mathbf{H}_{t}=$ $\mathbf{C}_{\Delta \mathbf{E}^{\prime}}=0$.

\section{Proposed Power Allocation Scheme}

In transmission over sub-channels $\overline{\mathbf{h}}_{i j, t}$, it can happen that we are faced with sub-channels that would require enormous transmit power to achieve acceptable bit error rates, especially in an imperfect CSI case. In this case it is beneficial, given the fixed amount of transmit power available, not to aim for equal error rates in all sub-channels, but perform an optimum power loading by minimizing the average bit error rate [25]. Some power allocation schemes are proposed in [26] to reduce the delay spread of the channel impulse response in the TR-MISOUWB systems. Authors in [26] did not consider imperfect CSI case and also ISI and MSI effects on system performance in which their analysis is true for the ideal and quasi-static channels. Therefore, based on previous findings such as [25] and [27], we propose a new power loading scheme for TRMIMO-UWB systems by minimizing the average bit error rate (BER) at the receiver in an imperfect CSI scenario and the time varying channels. This novel approach is a development of our previous power allocation work in the quasi-static MIMO channels [18]. The received signal at the MIMO channel output with TR pre-filtering and MMSE pre-equalizing in the time instant $t$, according to Fig. 1 can be written as

$$
\mathbf{y}_{t}=\left(\hat{\mathbf{H}}_{t}+\boldsymbol{\Delta} \mathbf{H}_{t}\right) \mathbf{H}_{T R, t} \mathbf{F}_{M M S E, t} \mathbf{x}_{t}+\mathbf{n}_{t}
$$

where $\Delta \mathbf{H}_{t}$ and $\mathbf{n}_{t}$ are assumed mutually independent and uncorrelated matrices. Also, $\mathbf{x}_{t}=$ $\frac{1}{\sqrt{N_{t}}}\left[x_{1, t}, x_{2, t}, \ldots, x_{N_{r}, t}\right]^{T}, \quad \mathbf{y}_{t}=\frac{1}{\sqrt{N_{r}}}\left[y_{1, t}, y_{2, t}, \ldots, y_{N_{r}, t}\right]^{T}$ and $\mathbf{n}_{t}=\left[n_{1, t}, n_{2, t}, \ldots, n_{N_{r}, t}\right]^{T}$ are the $N_{r} \times 1$ transmitted symbols, received symbols and AWGN vectors, respectively. The $N_{r}\left(2 L_{g}-1\right) \times N_{r}$ MMSE pre-equalizing matrix $\mathbf{F}_{M M S E, t}$ is given by (9). For simplicity of our analysis, we define the $N_{r}\left(2 L_{g}-1\right) \times 1$ MMSE pre-equalized transmitted symbols vector $\mathbf{x}_{t}^{\prime}$ as $\mathbf{x}_{t}^{\prime}=\mathbf{F}_{M M S E, t} \mathbf{x}_{t}$. Then, the received signal at $j^{t h}$ receive antenna in the time instant $t$ can be expressed by (12). The first part of the received signal is the desired data symbol. In this part, the equivalent channel is the autocorrelation of channels. Also, $v_{j, t}$ and $w_{j, t}$ are the interference from other symbols and the interference from the channel estimation error in the time instant $t$, respectively. In $v_{j, t}$ and $w_{j, t}$ parts, the equivalent channel is the crosscorrelation of channels. Therefore, the values of these terms are less than the former (first part of the received signal). Also, $v_{j, t}$ and $w_{j, t}$ terms in (12) appears as interference which degrades the performance of TR-MIMO-UWB system, especially in imperfect CSI. Some of this interference can be reduced by a MMSE pre-equalizer, but, we try to maximize the Signal to Interference plus Noise Ratio (SINR) more than ever by a power allocation scheme. If we define the conditional expectations as,

$$
\begin{aligned}
& E_{\hat{\mathbf{h}}_{i j, t} \mid \hat{\mathbf{h}}_{i j, t-\tau}}\left\{E_{\overline{\mathbf{H}}_{i j, t} \mid \overline{\mathbf{H}}_{i j, t-\tau}}\{.\}\right\}=E_{\text {con }}\{\cdot\} \\
& E_{\boldsymbol{\Delta} \mathbf{h}_{i j, t} \mid \boldsymbol{\Delta} \mathbf{h}_{i j, t-\tau}}\left\{E_{\overline{\mathbf{H}}_{i k, t} \mid \overline{\mathbf{H}}_{i k, t-\tau}}\{.\}\right\}=E_{\text {con-error }}\{.\}
\end{aligned}
$$

Then, the SINR at the $j^{\text {th }}$ receive antenna in the time instant $t$ is given by (14) in which $\|.\|_{F}$ denotes the Frobenius norm. By applying the above conditional expectations and using the approximate solution exploited for calculation of (9), also according to (3), (5) and (6), the SINR at the $j^{\text {th }}$ receive antenna in the time instant $t$ can be calculated as (15). If we define $\mathbf{R}_{i j, t-\tau}:=\hat{\mathbf{h}}_{i j, t-\tau} \overline{\mathbf{H}}_{i j, t-\tau}$ as auto-correlation vector of the channel vector $\hat{\mathbf{h}}_{i j, t-\tau}$ and, $\mathbf{C}_{i k, t-\tau}:={ }_{k \neq j} \hat{\mathbf{h}}_{i j, t-\tau} \overline{\mathbf{H}}_{i k, t-\tau}$ as cross-correlation vector of $\hat{\mathbf{h}}_{i j, t-\tau}$ with other sub-channels then, the SINR at the $j^{\text {th }}$ receive antenna is expressed as (16) in which the estimation errors of the different sub-channels are assumed to be mutually independent, i.e., $\boldsymbol{\Delta} \mathbf{h}_{i j, t} \amalg \boldsymbol{\Delta} \mathbf{h}_{m n, t}$ where $\amalg$ is standing for mutual independence. Performance of the TR-MIMO-UWB system depends on transceiver structure and the received signal properties, e.g., its probability density 


$$
y_{j, t}=\underbrace{\sum_{i=1}^{N_{t}} \hat{\mathbf{h}}_{i j, t} \overline{\mathbf{H}}_{i j, t} \mathbf{x}_{j, t}^{\prime}}_{\text {Signal }}+\underbrace{\sum_{v_{j, t}}^{N_{t}} \sum_{w_{j, t}}^{N_{r}} \hat{\mathbf{h}}_{i j, t} \overline{\mathbf{H}}_{i k, t} \mathbf{x}_{k, t}^{\prime}}_{\text {Interference }}+\underbrace{\sum_{i=1}^{N_{t}} \sum_{k=1}^{N_{r}} \Delta \mathbf{h}_{i j, t} \overline{\mathbf{H}}_{i k, t} \mathbf{x}_{k, t}^{\prime}}_{\text {i=1,kfj}}+\underbrace{n_{j, t}}_{\text {Noise }}
$$

$$
S I N R_{j, t}=\frac{E_{c o n}\left\{\left\|\sum_{i=1}^{N_{t}} \hat{\mathbf{h}}_{i j, t} \overline{\mathbf{H}}_{i j, t}\right\|_{F}^{2}\right\}}{E_{c o n}\left\{\left\|\sum_{i=1}^{N_{t}} \sum_{k=1, k \neq j}^{N_{r}} \hat{\mathbf{h}}_{i j, t} \overline{\mathbf{H}}_{i k, t}\right\|_{F}^{2}\right\}+E_{\text {con-error }}\left\{\left\|\sum_{i=1}^{N_{t}} \sum_{k=1}^{N_{r}} \Delta \mathbf{h}_{i j, t} \overline{\mathbf{H}}_{i k, t}\right\|_{F}^{2}\right\}+E\left\{\left\|\mathbf{n}_{j, t}\right\|_{F}^{2}\right\}}
$$

$$
S I N R_{j, t}=\frac{\rho^{4}\left(\left\|\sum_{i=1}^{N_{t}} \hat{\mathbf{h}}_{i j, t-\tau} \overline{\mathbf{H}}_{i j, t-\tau}\right\|_{F}^{2}\right)+\sigma_{h}^{2} \sigma_{h_{e q}}^{2}\left(1-\rho^{2}\right)^{2}}{\rho^{4}\left(\left\|\sum_{i=1}^{N_{t}} \sum_{k=1, k \neq j}^{N_{r}} \hat{\mathbf{h}}_{i j, t-\tau} \overline{\mathbf{H}}_{i k, t-\tau}\right\|_{F}^{2}+\left\|\sum_{i=1}^{N_{t}} \sum_{k=1}^{N_{r}} \boldsymbol{\Delta} \mathbf{h}_{i j, t-\tau} \overline{\mathbf{H}}_{i k, t-\tau}\right\|_{F}^{2}\right)+\sigma_{h_{e q}}^{2}\left(1-\rho^{2}\right)^{2}\left(\sigma_{h}^{2}+\sigma_{e}^{2}\right)+\sigma_{n}^{2}}
$$

$$
S I N R_{j, t}=\frac{\rho^{4}\left(\sum_{i=1}^{N_{t}} \sum_{l=0}^{2 L-2}\left|\mathbf{R}_{i j, t-\tau}(l)\right|^{2}\right)+\sigma_{h}^{2} \sigma_{h_{e q}}^{2}\left(1-\rho^{2}\right)^{2}}{\rho^{4}\left(\sum_{i=1}^{N_{t}} \sum_{k=1, k \neq j}^{N_{r}} \sum_{l=0}^{2 L-2}\left|\mathbf{C}_{i k, t-\tau}(l)\right|^{2}+L \sigma_{e}^{2} \sum_{i=1}^{N_{t}} \sum_{k=1}^{N_{r}} \sum_{l=0}^{L-1}\left|\hat{\mathbf{h}}_{i k, t-\tau}(l)\right|^{2}\right)+\sigma_{h_{e q}}^{2}\left(1-\rho^{2}\right)^{2}\left(\sigma_{h}^{2}+\sigma_{e}^{2}\right)+\sigma_{n}^{2}}
$$

function (PDF). For $L_{g} t_{s} \geq 5 \mathrm{nsec}$ where $t_{s}$ is the time resolution of the channel, the average numbers of paths is high, so using central limit theorem, the sum of a large number of independent, zero-mean random variables form a Gaussian PDF for the path gain [28]-[29]. But for $L_{g} t_{s}<5 n s e c$, path gain PDF is not Gaussian and as the $L_{g} t_{s}$ increases, the nonGaussian shape tends more to Gaussian, and the densities become more bell shaped [29]. According to the above discussion, the average BER of the TR-MIMO-UWB system at the $j^{\text {th }}$ receive antenna, assuming BPAM modulation, can be derived approximately from Eq. (16) as [27-29],

$$
B E R_{j, t} \approx \frac{1}{N_{t}} \sum_{i=1}^{N_{t}} Q\left(\sqrt{2 S I N R_{j, t} p_{i, j}}\right)
$$

where $p_{i, j}, P_{T, j}=\sum_{i=1}^{N_{t}} p_{i, j}$ and $Q($.$) are the transmit power$ assigned to $i^{\text {th }}$ transmit antenna, the total transmitted available power for the $j^{\text {th }}$ receive antenna and the Marcum Q-function, respectively. $Q(x)$ is defined as,

$$
Q(x)=\frac{1}{\sqrt{2 \pi}} \int_{x}^{+\infty} \exp \left(\frac{-t^{2}}{2}\right) d t
$$

For simplicity of our analysis we assume $\sigma_{\mathbf{x}}^{2}=1$, so that $P_{T, j}=N_{t}$. The optimum power allocation vector $\mathbf{P}_{j}=$ $\left[p_{1, j}, \cdots, p_{N_{t}, j}\right]^{T}$ that minimizes the average BER at the $j^{t h}$ receive antenna, when an imperfect CSI is presented, can be obtained by introducing the Lagrange function as [25],

$$
L_{G}=\frac{1}{N_{t}} \sum_{i=1}^{N_{t}} Q\left(\sqrt{2 S I N R_{j, t} p_{i, j}}\right)-\lambda\left(N_{t}-\sum_{i=1}^{N_{t}} p_{i, j}\right)
$$

With partial derivative we obtain,

$$
\frac{\partial L_{G}}{\partial p_{i, j}}=\frac{-1}{2 N_{t} \sqrt{\pi}} \sqrt{\frac{S I N R_{j, t}}{p_{i, j}}} \exp \left(-S I N R_{j, t} p_{i, j}\right)+\lambda
$$

Solving $\frac{\partial L_{G}}{\partial p_{i, j}}=0$ for $p_{i, j}$, we can allocate the power to each transmit antenna in a manner that,

$$
\left(\frac{1}{S I N R_{j, t}}\right) p_{i, j} \exp \left(S I N R_{j, t} p_{i, j}\right)=\frac{1}{4 N_{t}^{2} \lambda^{2} \pi}
$$

and finally,

$$
p_{i, j}(\lambda)=\left(\frac{1}{S I N R_{j, t}}\right) W\left(\left(S I N R_{j, t}\right)^{2} \frac{1}{4 N_{t}^{2} \lambda^{2} \pi}\right)
$$

where $W($.$) is the real valued Lambert's W-function defined$ as the inverse of the function $f(x)=x \exp (x)$ for $x \geq 0$, i.e., $W(x)=a \Leftrightarrow a \exp (a)=x$ [30]. Since the $W(x)$ function is real and concave, the unique solution for power allocation vector to minimize the average BER at the $j^{\text {th }}$ receive antenna can be found by the following simple iterative procedure [31]. 1. Choose a positive $\lambda$, which fulfils,

$$
\left(S I N R_{j, t}\right)\left(\frac{1}{4 N_{t}^{2} \lambda^{2} \pi}\right) \leq P_{T, j}
$$

2. Calculate,

$$
\hat{P}_{T, j}=\left(\frac{1}{S I N R_{j, t}}\right) W\left(\left(S I N R_{j, t}\right)^{2} \frac{1}{4 N_{t}^{2} \lambda^{2} \pi}\right)
$$

3. If $\hat{P}_{T, j}$ is not yet sufficiently close to $P_{T, j}$ then multiply $\lambda$ by $\frac{P_{T, j}}{\hat{P}_{T, j}}$ and go back to step (2).

4. Compute $\mathbf{P}_{j}=\left[p_{1, j}, \cdots, p_{N_{t}, j}\right]^{T}$ according to (22).

Note that since $W(x)$ for $x>\frac{-1}{e}$ is a monotonic function then according to (22) the highest powers $\left(\max p_{i, j}\right)$ are assigned to the weakest signals so the SINR values approximately stay constant for all sub-channels. The above algorithm should be iterated for all of $N_{r}$ receive antenna and then, we achieve the total optimized power allocation vector as $\mathbf{P}=\sum_{j=1}^{N_{r}} \mathbf{P}_{j}$. Note that the total available power for data transmission is $P_{T}=\frac{1}{N_{r}} \sum_{j=1}^{N_{r}} P_{T, j}$. 


\section{Simulation Results and Discussion}

To evaluate the performance of the proposed solutions for the TR-MIMO-UWB system, Monte Carlo simulations are conducted in this section. As in [11], the second-order derivative of Gaussian pulse has been used as the transmitted pulse $p(t)$, which is mathematically defined as,

$$
p(t)=\left(1-4 \pi\left(\frac{t-t_{c}}{T_{p}}\right)^{2}\right) \exp \left(-2 \pi\left(\frac{t-t_{c}}{T_{p}}\right)^{2}\right)
$$

where $T_{p}$ is a parameter corresponding to pulse width, and $t_{c}$ is a time shifting of the pulse. In the following simulations, we consider $T_{p}=5 n s e c$, and $t_{c}=2.5 n s e c$. Also, one pulse per symbol is assumed, i.e., symbol duration $T$ is assumed $5 \mathrm{nsec}$ where is equal to transmission rate of 200Mbps and also, sampling time $t_{c}=0.167 n s e c$ is considered. We assume that the signal is transmitted over UWB channel. As in [11], we use the IEEE 802.15.3a CM4 channel model [32]-[33] as a worst case for each channel realization in simulations. Although the channel model CM4 is designed for Single-Input Single-Output (SISO) scenario, the extension to a MIMO configuration is achieved by assuming that the MIMO channel parameters are independent and identically distributed realizations from the same statistical model. We further consider the case of a $2 \times 2$ TR-MIMO-UWB system set-up with BPAM modulated data streams. In our simulations, we use the normalized Doppler frequency $f_{d} T=0.001$, $f_{d} T=0.005$ and $f_{d} T=0.01$. Also, the channel estimation error variance $\sigma_{e}^{2}=0.1$ is assumed. At first, it is necessary that we clarify some phrases mentioned in some simulation results. Phrase "Perfect CSI" means the conventional TRMIMO-UWB system in perfect CSI. Clearly, this scenario does not need to the optimization schemes. We provide a benchmark curve according to [11]. Phrase "Conventional TRMIMO-UWB" means the time reversal based MIMO-UWB system in an imperfect CSI without any optimization. Also, "proposed MMSE TR-MIMO-UWB" means the application of the conventional TR (time reversal based MIMO-UWB system in an imperfect CSI) with the MMSE optimized pre-filter. Phrase "Proposed with loading" means the application of the proposed MMSE TR-MIMO-UWB with the power allocation scheme. Finally, "Proposed without loading" is referred to the proposed MMSE TR-MIMO-UWB.

To optimize the TR-MIMO-UWB system performance of Fig. 1 and to mitigate the imperfect CSI effects, we use the proposed robust MMSE solution mentioned in Section 3. Figs. 2 and 3 plot the average BER performances of the $2 \times 2$ TR-MIMO-UWB system with proposed optimization for $N=5,10$, respectively. Clearly, it can be seen from Figs. 2 and 3 that, our robust MMSE solution exhibits a lower average BER performance when compared to the conventional TR-MIMO-UWB system design. More specifically, from the figures, it can be observed that the proposed method have more advantage for shorter $N$ where the channel time correlation is noticeable, in that, as $N$ increases, the correlation coefficient $\rho=J_{0}\left(2 \pi f_{d} \tau\right)=J_{0}\left(2 \pi f_{d} N T\right)$ decreases and so, the optimized solutions performance is diminished.

Figs. 4 and 5 plot the average BER performances of the
$2 \times 2$ TR-MIMO-UWB system with proposed optimization for $f_{d} T=0.001$ and $f_{d} T=0.005$, respectively. As it can be observed from Figs. 4 and 5 that, for lower normalized Doppler frequency, our proposed method has good performance over all values of $N$ while in the higher normalized Doppler frequency, the performance is noticeable for shorter $N \mathrm{~s}$ where the channel time correlation is noticeable.

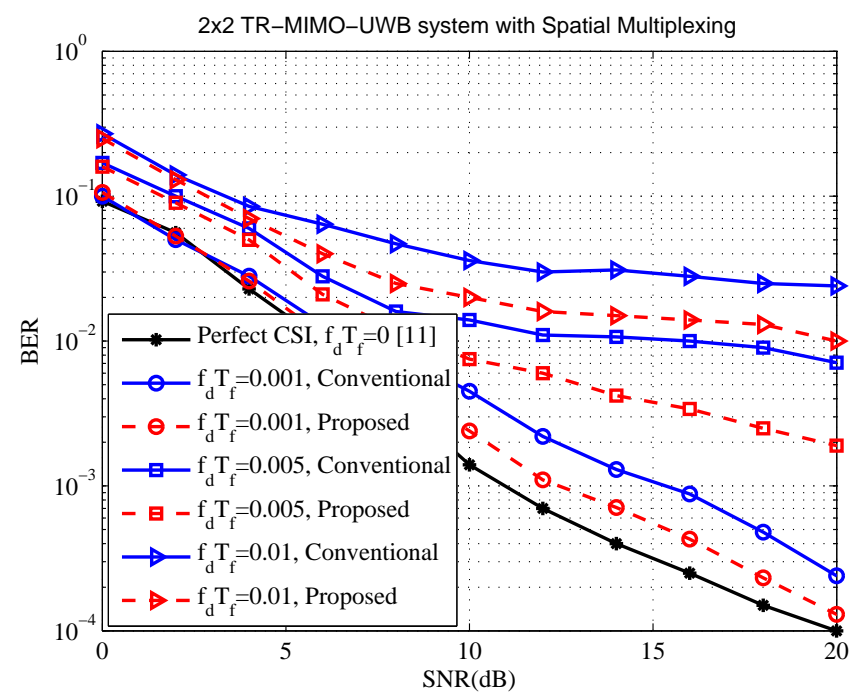

Fig. 2. BER performance of the $2 \times 2$ TR-MIMO-UWB system with proposed MMSE optimization for $N=5, \sigma_{e}^{2}=0.1$ and different Doppler values.

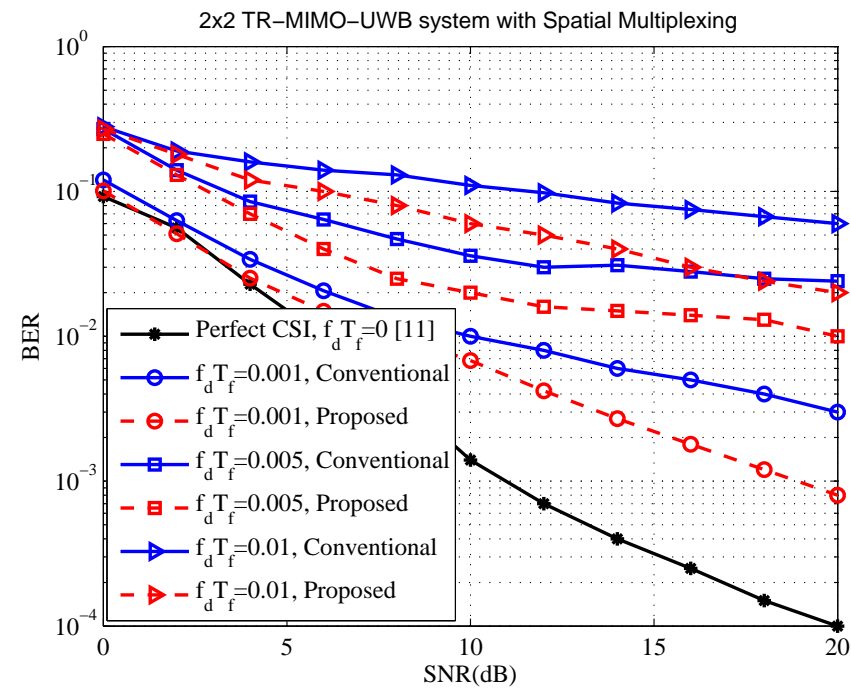

Fig. 3. BER performance of the $2 \times 2$ TR-MIMO-UWB system with proposed MMSE optimization for $N=10, \sigma_{e}^{2}=0.1$ and different Doppler values.

In addition to the MMSE robust optimization scheme, we use the proposed power loading solution mentioned in Section IV to improve the TR-MIMO-UWB system performance more than ever. In Figs. 6-9, we can observe the performance of the proposed power loading for the MMSE robust optimization. As can be seen from these figures, the performance of power 
loading is noticeable, especially for high SNR values. Such as, according to Figs. 6-7, for $f_{d} T=0.001$, the power loading scheme substantially outperforms the robust optimization method about $2 \mathrm{~dB}$ SNR in Fig. 6 and $4 \mathrm{~dB}$ SNR in Fig. 7 both at the average $\mathrm{BER}=10^{-3}$. Also, according to Figs. 8-9, the power loading scheme can be used to increase the TRMIMO-UWB system performance, considerably. Furthermore, the system performance is mitigated as $N$ increases. Therefore, our proposed method significantly depends on the values of $N$ or the time-correlation coefficient $\rho$.

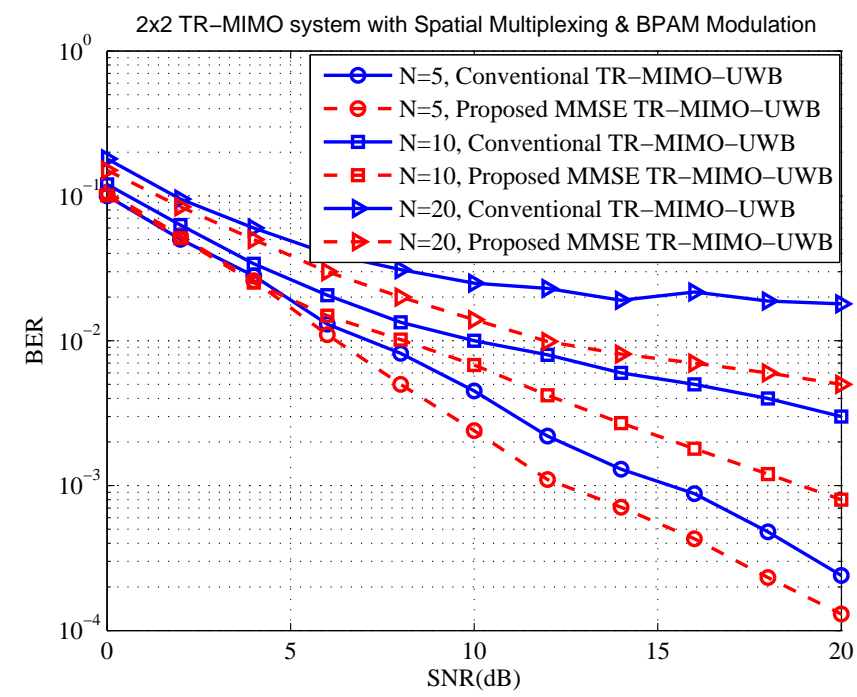

Fig. 4. BER performance of the $2 \times 2$ TR-MIMO-UWB system with proposed MMSE optimization for $f_{d} T=0.001, \sigma_{e}^{2}=0.1$ and different $N$ values.

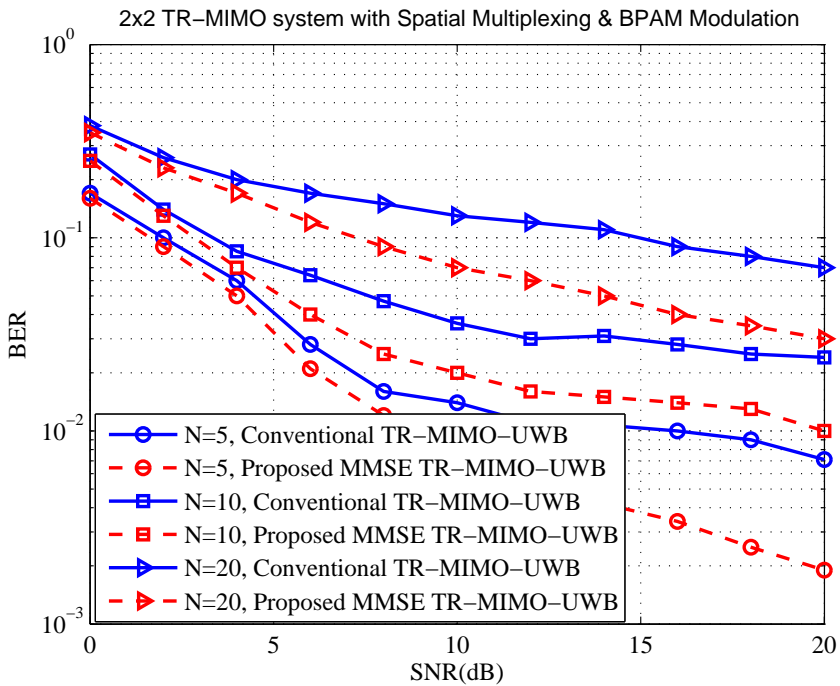

Fig. 5. BER performance of the $2 \times 2$ TR-MIMO-UWB system with proposed MMSE optimization for $f_{d} T=0.005, \sigma_{e}^{2}=0.1$ and different $N$ values.

As we observed from the simulation results, we will confront the weak performance of our methods as the speed of the user or vehicle increases, in that by increasing $f_{d}$,

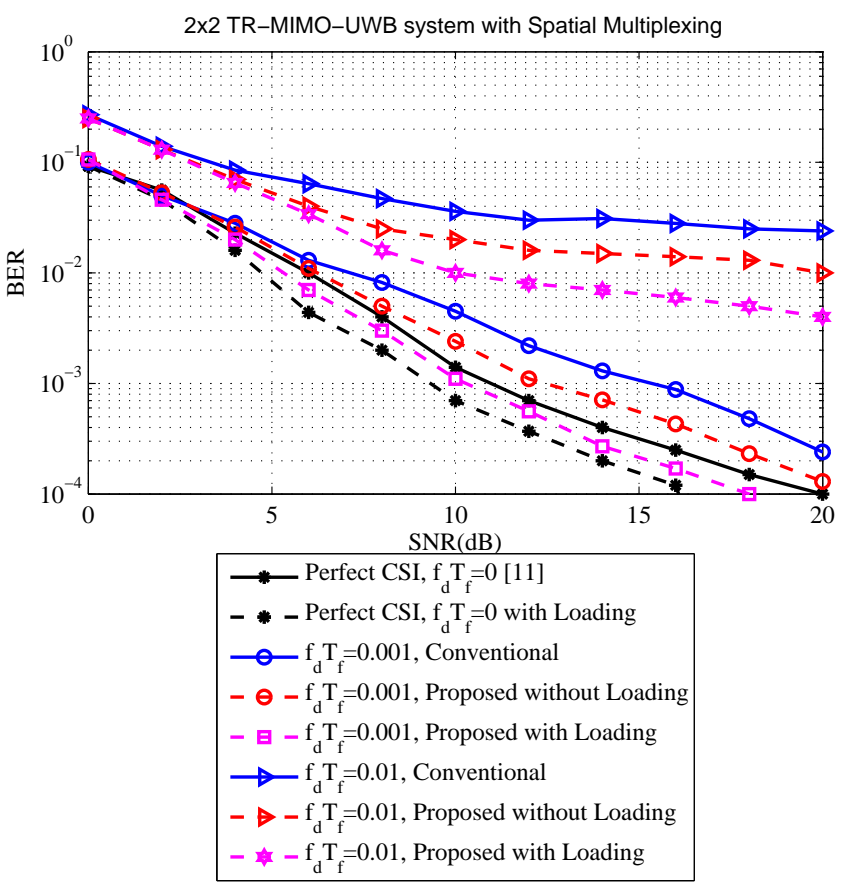

Fig. 6. BER performance of the $2 \times 2$ TR-MIMO-UWB system with proposed MMSE optimization and power loading for $N=5$, $\sigma_{e}^{2}=0.1$ and different Doppler values.

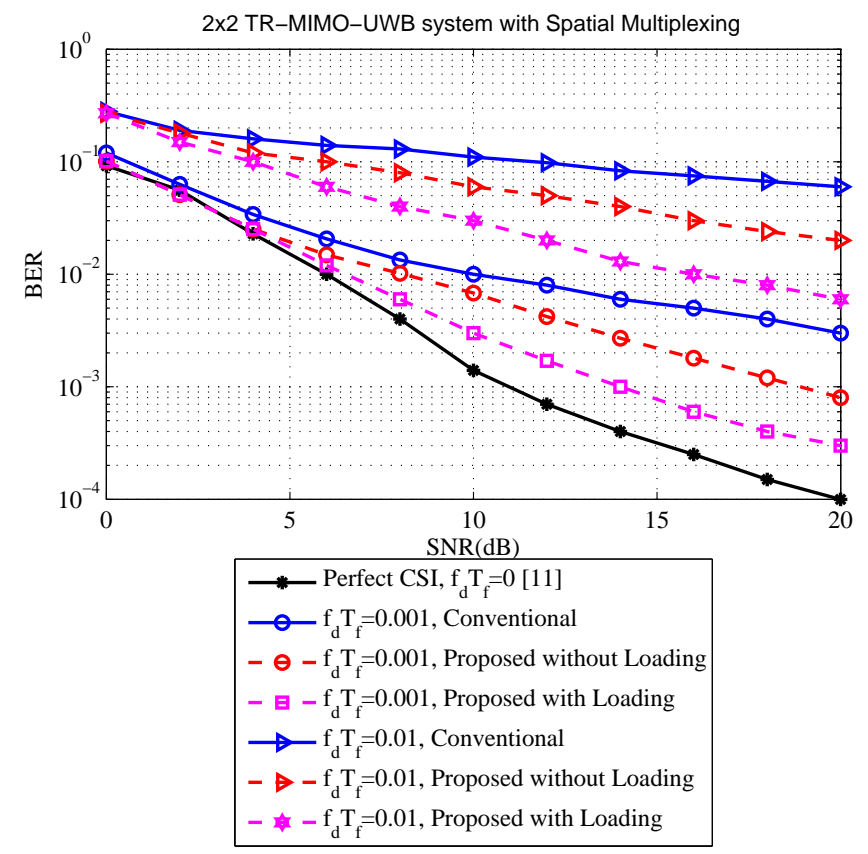

Fig. 7. BER performance of the $2 \times 2$ TR-MIMO-UWB system with proposed MMSE optimization and power loading for $N=10$, $\sigma_{e}^{2}=0.1$ and different Doppler values.

the correlation coefficient decreases (it depends by zero-order Bessel function) and so, SINR decreases. As a result, the 


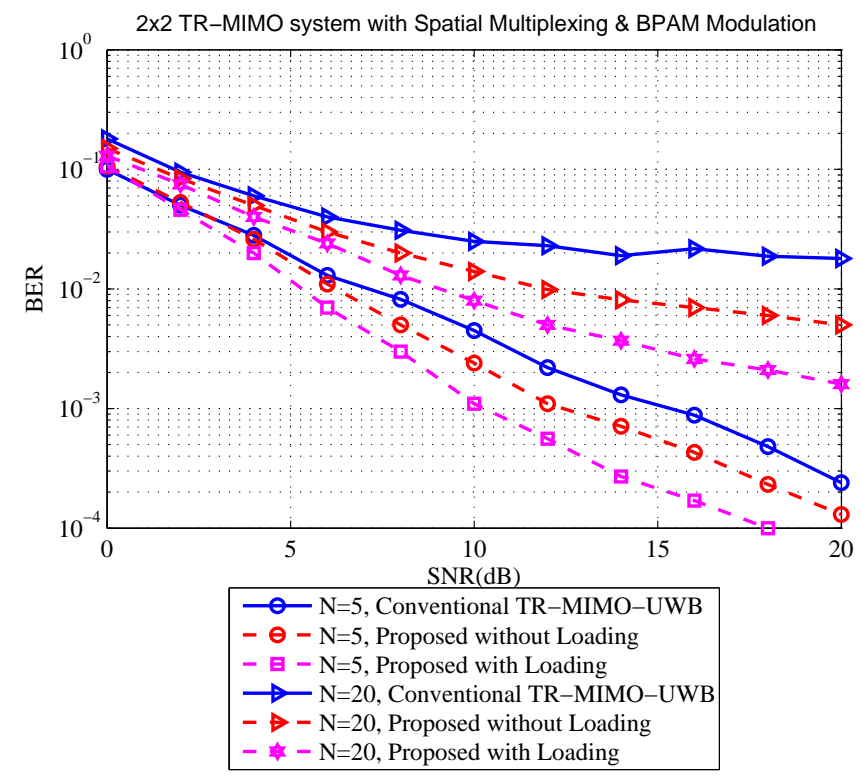

Fig. 8. BER performance of the $2 \times 2$ TR-MIMO-UWB system with proposed MMSE optimization and power loading for $f_{d} T=0.001$, $\sigma_{e}^{2}=0.1$ and different $N$ values.

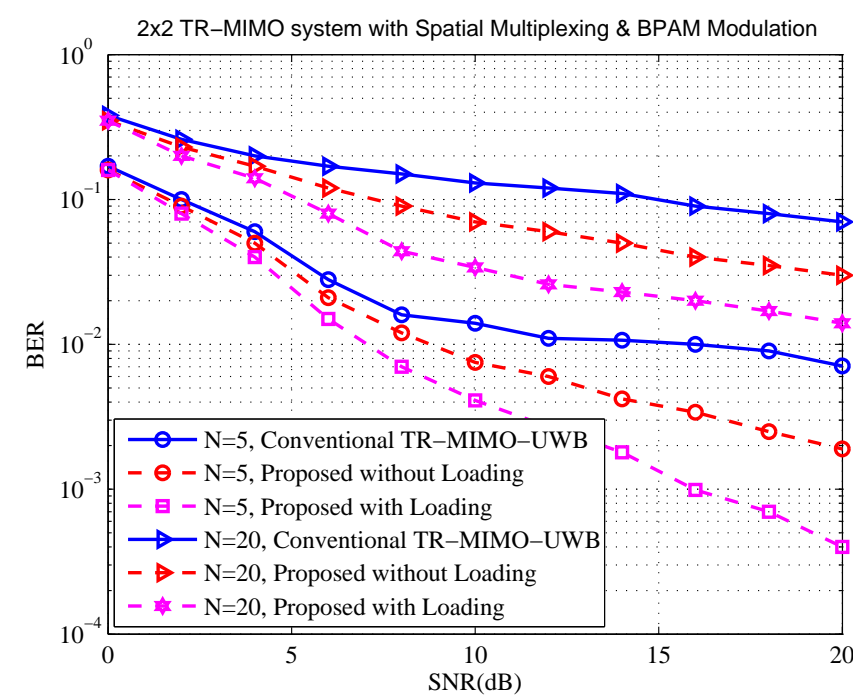

Fig. 9. BER performance of the $2 \times 2$ TR-MIMO-UWB system with proposed MMSE optimization and power loading for $f_{d} T=0.005$, $\sigma_{e}^{2}=0.1$ and different $N$ values.

BER increases. Similar discussions can be presented for $N$. Therefore, the best performance of our solutions is in lower speed of user or lower $N$ values.

\section{CONCLUSiOnS}

A robust MMSE TR-MIMO-UWB system design that exploits the knowledge of the conditional channel distribution, given the available outdated CSI in $\tau$ second ago was proposed in this paper. In proposal solution, once the channel estimation error covariance matrix $\mathbf{C}_{\boldsymbol{\Delta} \mathbf{H}_{t}}$, outdated CSI and the time correlation coefficient $\rho$ is transmitted from the receiver to the transmitter in the beginning of a data block and then, they are used during that block. This solution mitigates the feedback overhead in time varying channel significantly than traditional method in which we used to feedback the CSI during a data block. We have also shown that our robust approach outperforms the traditional design in terms of BER plots in time varying scenario, especially for high SNR and shorter data block length where the time correlation of the channel is noticeable. Then, the power loading strategy was proposed for the imperfect CSI in the robust optimized MMSE TR-MIMO-UWB system. It was observed that it brought a performance gain in the $2 \times 2$ TR-MIMO-UWB system in an imperfect CSI scenario and the robust optimized system.

\section{REFERENCES}

[1] F. Nekoogar, Ultra-wideband communications: fundamentals and applications, Pearson Education, First printing, September, 2005.

[2] J. H. Reed, An Introduction to Ultra Wideband Communication Systems, Prentice Hall PTR, April 05, 2005.

[3] R. Hoctor and H. Tomlinson, Delay-Hopped Transmitted-Reference $R F$ Communications, Proc. IEEE 2nd Ultra Wideband Systems and Technologies (UWBST02), Baltimore, MD, pp. 265-269, May 2002.

[4] R. J. Fontana, E. Richley, and J. Barney, Commercialization of an Ultra Wideband Precision Asset Location System, Proc. IEEE Conference UWB systems and Technologies, Reston, VA, 2003.

[5] J. D. Choi and W. E. Stark, Performance of Ultra-Wideband Communications with Suboptimal Receivers in Multipath Channels, IEEE Journal on Selected Areas in Communications, vol. 20, pp. 1754-1766, Dec. 2002.

[6] K. Witrisal, G. Leus, G. J. M. Janssen et al., Noncoherent ultrawideband systems: an overview of recent research activities, IEEE Signal Processing Magazine, vol. 26, no. 4, pp. 4866, 2009.

[7] J. Zhang, P. V. Orlik, Z. Sahinoglu, A. F. Molisch, and P. Kinney, UWB systems for wireless sensor networks, Proceedings of the IEEE, vol. 97 no. 2, pp. 313331, 2009.

[8] M. Fink, Time-reversed acoustic, Scientific Amer., pp. 67-73, Nov.1999.

[9] G. Foschini and M. Gans, On limits of wireless communications in a fading environment when using multiple antennas, Wireless Pers. Commun, 1998, 6, (3), pp.311-335.

[10] T. Kaiser and F. Zheng, Ultra Wideband Systems with MIMO, John Wiley Sons Ltd.,UK, 2010.

[11] H. Nguyen, F. Zheng and T. Kaiser, Antenna selection for time reversal MIMO UWB systems, In IEEE 69th Vehicular Technology Conf., Barcelona, Spain, 2629 April 2009.

[12] T. Strohmer, M. Emami, J. Hansen, G. Papanicolaou, and A. J. Paulraj, Application of time reversal with MMSE equalizer to UWB Communications, Proc. IEEE Global Telecommunications Conference, Dallas, Texas, Nov.29-Dec.3, 2004, pp.3123-3127.

[13] T. Wang, and T. Lv, Canceling Interferences for High Data Rate Time Reversal MIMO UWB System: A Precoding Approach, EURASIP Journal on Wireless Communications and Networking, vol. 2011, pp.110 .

[14] T. K. Nguyen, H. Nguyen, F. Zheng, and T. Kaiser, Spatial correlation in SM-MIMO-UWB systems using a pre-equalizer and pre-Rake filter, in Proceedings of the IEEE International Conference on Ultra-Wideband (ICUWB '10), pp. 540543, September 2010.

[15] H. Nguyen, V. D. Nguyen, T. K. Nguyen, K. Maichalernnukul, F. Zheng, and T. Kaiser, On the Performance of the Time Reversal SM-MIMO UWB System on Correlated Channels, International Journal of Antennas and Propagation, , doi:10.1155/2012/929018, Volume 2012.

[16] H. Khaleghi Bizaki, S. Alizadeh, Mitigation of Channel Estimation Error in TR-UWB system Based on a Novel MMSE Equalizer, Springer Annals of Telecommunication, DOI 10.1007/s12243-012-0325-8, published online: 6 Oct. 2012.

[17] S. Alizadeh, H. Khaleghi Bizaki and M. Okhovvat, Effect of Channel Estimation Error on Performance of Time Reversal-UWB Communication System and its Compensation by Pre-filter, IET Communications, vol. 6, no. 12, pp.17811794, Nov. 2012. 
[18] S. Alizadeh, and H. Khaleghi Bizaki, Novel Robust Optimization and Power Allocation of Time Reversal-MIMO-UWB Systems in an Imperfect CSI, The International Journal of Advances in Telecommunications, Electrotechnics, Signals and Systems (IJATES2), Vol. 2, No. 2 (2013), pp. 84-91.

[19] S. Alizadeh, and H. Khaleghi Bizaki, An Enhanced MMSE based Pre-processing Scheme for Time Reversal MIMO-UWB Systems in an Imperfect CSI, Proceedings of the 21st IEEE Iranian Conference on Electrical Engineering (ICEE’2013), Mashhad, Iran, doi: 10.1109/IranianCEE.2013.6599663, 14-16 May 2013.

[20] I. H. Naqvi, P. Besnier and G. El Zein, Robustness of a time-reversal ultra wideband system in non-stationary channel environment, IET Microwaves, Antennas and Propagation, March 2011, Vol. 5, No. 4, pp. $468-475$

[21] X. Liu, B. Z. Wang, S. Xiao and S. Lai, Post-Time-Reversed MIMO Ultra wideband Transmission Scheme, IEEE Transactions on Antennas and Propagation, May 2010, vol. 58, no.5, pp.1731-1738.

[22] D. Abbasi-Moghadam, V. T. Vakili, Effect of Channel Estimation Error on Time Reversal UWB Communication System, Springer Wireless Personal Communications, DOI: 10.1007/s11277-011-0460-y, published online: 25 Nov. 2011

[23] H. Khaleghi Bizaki, Tomlinson-Harashima Precoding Optimization over Correlated MIMO Channels, IEEE International Conference on Wireless Communications, Networking, and Information Security (WCNIS), pp. 207-211, Jun. 2010.

[24] B. Hassibi and B. M. Hochwald, How much training is needed in multiple antenna wireless links?, IEEE Trans. Inf. Theory, vol. 49, no. 4, pp. 951-963, Apr. 2003.

[25] C. Windpassinger, Detection and Precoding for Multiple Input Multiple Output Channels, PhD dissertation, Erlangen University, Jan. 2004

[26] P. Kyristi, G. Panicolaou, and A. Oprea, MISO time reversal and 918 delay-spread compression for FWA channels at $5 \mathrm{GHz}$, IEEE Antennas 919 Wireless Propag. Lett., Dec. 2004, vol. 3, pp. 9699.

[27] H. K. Bizaki and A. Falahati, Tomlinson-Harashima precoding with imperfect channel state information, IET. Communication journal, vol.2, no.1, pp.151-158, January 2008.

[28] K. Witrisal, Statistical analysis of the IEEE 802.15.4a UWB PHY over Multipath Channels, IEEE Wireless Communications and Networking Conference, WCNC2008, March 31April 32008.

[29] D. Abbasi-moghadam, V. Tabataba Vakili, Characterization of Indoor Time Reversal UWB Communication Systems: Spatial, Temporal and Frequency Properties, Wiley International Journal of Communication Systems, doi:10.1002/dac.1140, published online 28 April 2010.

[30] R. M. Corless, G. H. Gonnet, D. E. G. Hare, D. J. Jeffrey, and D. E. Knuth, On the Lambert $W$ Function, Advances in Computational Mathematics, volume 5, 1996, pp. 329-359.

[31] T. Hunziker, D. Dahlhaus, Optimal Power Adaptation for OFDM Systems with Ideal Bit-Interleaving and Hard-Decision Decoding, IEEE International Conference on Communications (ICC), vol. 5, 2003, pp:3392-3397.

[32] J. Foerster et al., Channel modeling sub-committee report final, IEEE P802.15 Wireless Personal Area Networks, P802.15-02/490r1-SG3a, Feb. 2003.

[33] A. F. Molisch, K. Balakrishnan, D. Cassioli, C. Chong, S. Emami, A Fort, J. Karedal, J. Kunisch, H. Schantz, U. Schuster, and K. Siwiak, IEEE 802.15.4a Channel model final report, Tech. Rep., 2004.

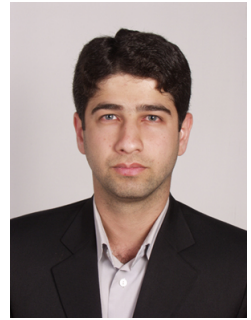

Sajjad Alizadeh was born in Roudsar, Guilan Province, Iran on November 3, 1983. He received the Associate Degree (A.D.) from Electronics Engineering College (Electronics Industry University) of Shiraz University (Shiraz University of Technology (SUTECH) today), Shiraz, Iran, in 2005 and the B.Sc. degree from Shahid Rajaee Teacher Training University (SRTTU), Tehran, Iran, in 2007, both in electronics. Also he received the M.Sc. degree in Communication Engineering from Imam Hossein University (IHU), Tehran, Iran, in 2011. He is currently working toward the Ph.D. degree in Electrical Engineering (Communication Systems) at Yazd University, Yazd, Iran. He has served as a referee in IET Communications journal. His current research interests are in the area of UWB wireless communications with special emphasis on digital and statistical signal processing, Time Reversal signaling, propagation channels modeling, multiple-antenna (MIMO) schemes, Cognitive Radio networks, Detection and Estimation theory, Convex Optimizations and Interference Modeling in Wireless Communications.

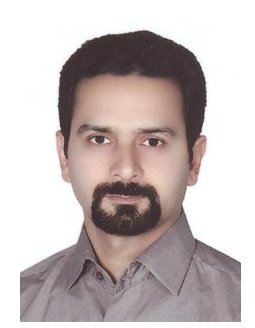

Hossein Khaleghi Bizaki received his Ph.D. degree in Electrical Engineering, Communication system, from Iran University of Science and Technology (IUST), Tehran, Iran, in 2008. Dr. Bizaki started his work as assistant professor in 2008. Now, he is author or co-author of more than 30 publications. His research interests include Information Theory, Coding Theory, Wireless Communication, MIMO Systems, Space Time Processing, and other topics on Communication System and Signal Processing.

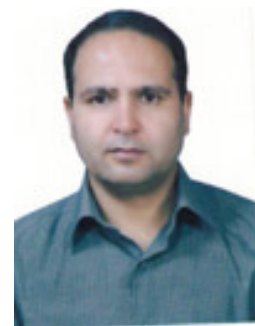

Reza Saadat (M'11) received the B.Sc. degree in electronics engineering, the M.Sc. degree in communication systems engineering, and the Ph.D. degree in electrical engineering from Isfahan University of Technology, Isfahan, Iran, in 1992, 1996, and 1999, respectively. Since 1999, he has been an Assistant Professor with the Department of Electrical and Computer Engineering, Yazd University, Yazd, Iran. His research interests include received signal strength positioning, resource allocation, and wireless sensor networks. 